\title{
Modeling Lepton-Nucleon Inelastic Scattering from High to Low Momentum Transfer
}

\author{
S. Alekhin*, S.A. Kulagin ${ }^{\dagger}$ and R. Petti** \\ *Institute for High Energy Physics, 142281 Protvino, Moscow region, Russia \\ ${ }^{\dagger}$ Institute for Nuclear Research, 117312 Moscow, Russia \\ ${ }^{* *}$ Depertment of Physics and Astronomy, University of South Carolina, Columbia SC 29208, USA
}

\begin{abstract}
.
We present a model for inclusive charged lepton-nucleon and (anti)neutrino-nucleon cross sections at momentum transfer squared, $Q^{2}, \sim 1 \mathrm{GeV}^{2}$. We quantify the impact of existing low-Q charged-lepton deep-inelastic scattering (DIS) data on effects due to high-twist operators and on the extraction of parton distribution functions (PDFs). No evidence is found for twist- 6 contributions to structure functions (SF), and for a twist-4 term in the logitudinal SF at $x \gtrsim 0.1$. We find that DIS data are consistent with the NNLO QCD approximation with the target mass and phenomenological high twist corrections. For $Q^{2}<1 \mathrm{GeV}^{2}$, we extend extrapolation of the operator product expansion, preserving the low- $Q$ current-conservation theorems. The procedure yields a good description of data down to $Q^{2} \sim 0.5 \mathrm{GeV}^{2}$. An updated set of PDFs with reduced uncertainty and applicable down to small momentum transfers in the lepton-nucleon scattering is obtained.
\end{abstract}

Keywords: Neutrino cross-sections, high twists, structure functions, parton distribution functions PACS: $13.15 .+\mathrm{g}$ 13.60.-r

\section{INTRODUCTION}

At high momentum transfer $Q$ the lepton-nucleon cross-sections are well described in terms of parton distributions (PDFs), whose $Q^{2}$ evolution is well-understood in perturbative Quantum Chromo-Dynamics (QCD). The universality of the partonic description allows to obtain predictions for a variety of probes $(e, \mu, v, \bar{v})$ and targets, which have been extensively verified by experiments. However, by lowering progressively $Q$ nonperturbative phenomena become more and more important for a precise modeling of cross-sections.

The existing DIS data at small momentum transfer $Q$ in principle can shed light on the interplay between perturbative and non-perturbative phenomena and clarify the limits of applicability of the parton model. Furthermore they can put valuable constraints on the parton distribution functions (PDFs) and on the strong coupling constant $\alpha_{\mathrm{s}}$, due to their excellent statistical precision. However, significant high-order QCD corrections are required to study such kinematical region. The recent progress in the NNLO QCD calculations [1] allows to use the DIS data down to $Q \sim 1 \mathrm{GeV}$ in global QCD fits by keeping the perturbative stability under control. 
In the formalism of the Operator Product Expansion (OPE) unpolarized structure functions can be expressed in terms of powers of $1 / Q^{2}$ (power corrections):

$$
F_{2, T, 3}\left(x, Q^{2}\right)=F_{2, T, 3}^{\tau=2}\left(x, Q^{2}\right)+\frac{H_{2, T, 3}^{\tau=4}(x)}{Q^{2}}+\frac{H_{2, T, 3}^{\tau=6}(x)}{Q^{4}}+\ldots .
$$

The first term $(\tau=2)$, expressed in terms of PDFs, represents the Leading Twist (HT) describing the scattering off a free quark and is responsible for the scaling of SF via perturbative QCD $\alpha_{S}\left(Q^{2}\right)$ corrections. The Higher Twist (HT) terms $(\tau=4,6)$ reflect instead the strength of multi-parton correlations (qq and qg). Since such corrections spoil factorization one has to consider their impact on the PDFs extracted in the analysis of low- $Q$ data. Due to their non-perturbative origin, current models can only provide a qualitative description for such contributions, which must then be determined phenomenologically from data.

Existing information about high twist terms in lepton-nucleon structure functions is scarce and somewhat controversial. Early analyses [2, 3] suggested a significant HT contribution to the longitudinal SF $F_{L}$. The subsequent studies with both charged leptons [4, 5, 6] and neutrinos [7] raised the question of a possible dependence on the order of QCD calculation used for the leding twist. The common wisdom is generally that HTs only affect the region of $Q^{2} \sim 1 \div 3 \mathrm{GeV}^{2}$ and can be neglected in the extraction of the leading twist.

In this communication we report our results on using the DIS data down to $Q=1 \mathrm{GeV}$ in the global QCD fit of PDFs with power corrections included in the analysis.

\section{PROCEDURE}

The analyzed data set consist of the world charged-leptons DIS cross section data for the proton and deuteron targets by the SLAC, BCDMS, NMC, FNAL-E-665, H1, and ZEUS experiments supplemented by the fixed-target Drell-Yan data, the latter constrain the sea quark distribution, which is poorly determined by the DIS data alone. Basically the same combination of data was used in the earlier fit of Ref.[9] with the cut $Q^{2}>$ $2.5 \mathrm{GeV}^{2}$ imposed on the DIS data. In the present fit alongside with the softer cut imposed on the SLAC and NMC data, $Q>1 \mathrm{GeV}$, we also add the DIS data by FNAL-E-665 experiment [10] since they give additional constraint on the PDFs at small $x$ provided not too stringent cut on $Q$ is applied. The cut on invariant mass of the hadron system $W>1.8 \mathrm{GeV}$ is imposed on the DIS data to avoid the resonance region. The total number of data points (NDP) used in the fit is 3076, in the range of $x=0.0001 \div 0.9$. The analysis 1 is performed in the NNLO QCD approximation with the target mass corrections [8] taken into account and the dynamical twist-4 (twist-6) terms parameterized in the additive form as model independent spline functions $H(x)$. Deuteron data are corrected for nuclear effects following the model [11].

\footnotetext{
${ }^{1}$ Details of the theoretical ansatz can be found in Ref.[9].
} 

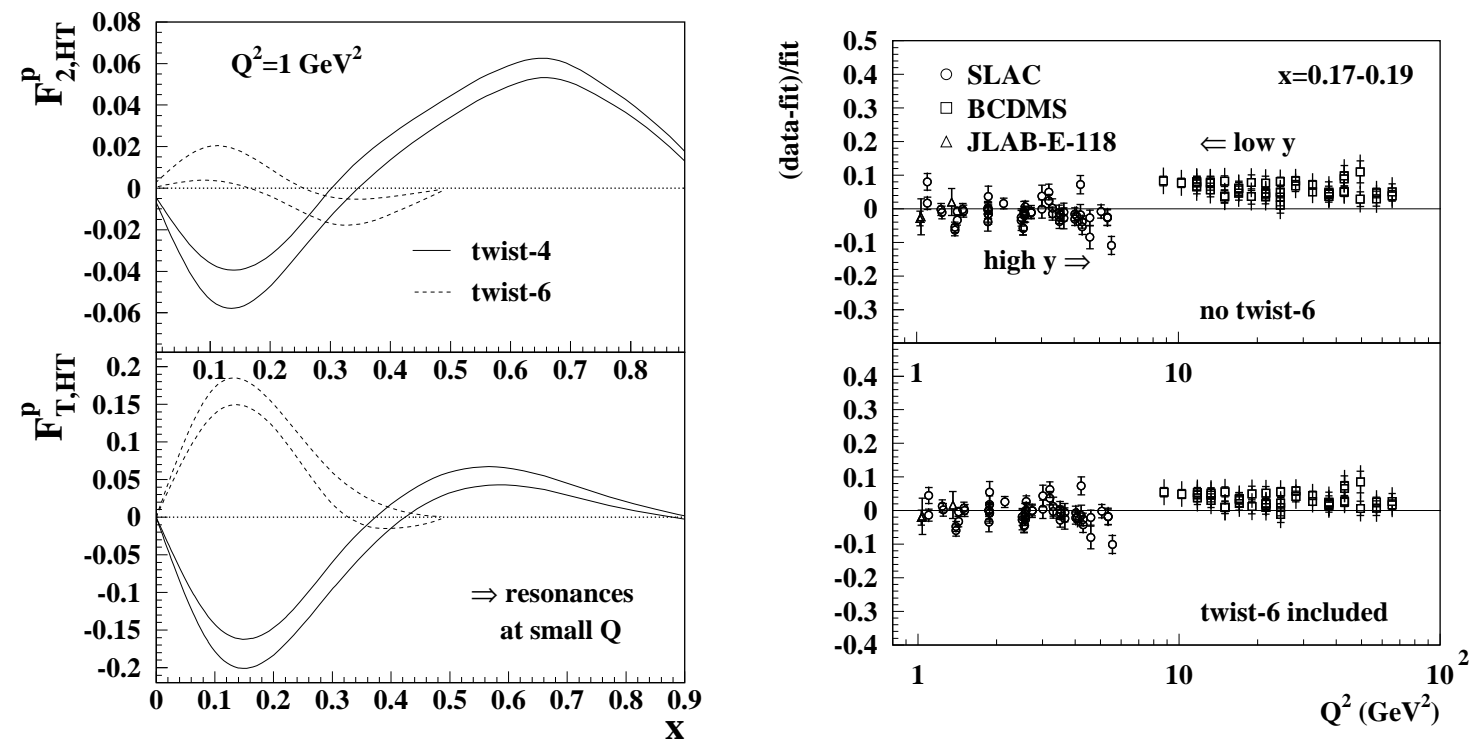

FIGURE 1. Left figure: the $1 \sigma$ error bands for the twist- 4 (solid lines) and twist- 6 (dashes) terms in the proton $F_{2}$ (upper panel) and $F_{\mathrm{T}}$ (lower panel). The arrow indicates the region of $x$ with the limited potential for the determination of twist- 6 terms due to the cut on $W$. Right figure: pulls corresponding to the fits with and without twist- 6 terms. The arrows in the upper panel indicate the high- $y$ and low- $y$ regions for the SLAC and BCDMS data. The data points for the JLAB-E-118 experiment at $Q \sim 1 \mathrm{GeV}$ were not used in the fit.

In addition, the recent neutrino and antineutrino cross-section data from the CHORUS experiment [12] are added to the global fit for $Q>1.0 \mathrm{GeV}$ and $x \geq 0.045$, mainly to constrain the corresponding HT terms.

\section{DETERMINATION OF HIGH TWIST TERMS}

As a first step of the analysis we checked possible twist- 6 contributions to the DIS structure functions by keeping the terms $H^{\tau=6}(x)$ in Eq.(1). Due to the $W$ cut, twist6 terms are insensitive to the large- $x$ data and therefore we set them to zero at $x \geq 0.5$. Values of the HT terms at $x=0$ were also set to zero in view of the fact no clear evidence of saturation effects was found in the small $x$ HERA data.

Figure 1 (left) shows the HT terms in $F_{2, \mathrm{~T}}$ obtained in such a variant of our fit. Surprisingly, we observe a large positive contribution from the twist- 6 term to $F_{\mathrm{T}}$ at $x \sim 0.15$. At the same time this contribution is compensated by a corresponding negative twist- 4 term. Since the twist- 4 and twist- 6 coefficients are similar in magnitude but opposite in sign, the overall sum of HT terms demonstrate a weak dependence on $Q$. This observation leads us to the conclusion that in fact the twist- 6 term in $F_{\mathrm{T}}$ absorbs some non-power-like effects.

When we remove twist- 6 terms from our fits the magnitude of the pulls appears to be maximal not at the lowest $Q$ values, but at $Q^{2} \sim 7 \mathrm{GeV}^{2}$, exactly in the region of overlap between SLAC and BCDMS data (see Fig.1 right). Indeed, these two data sets show a 

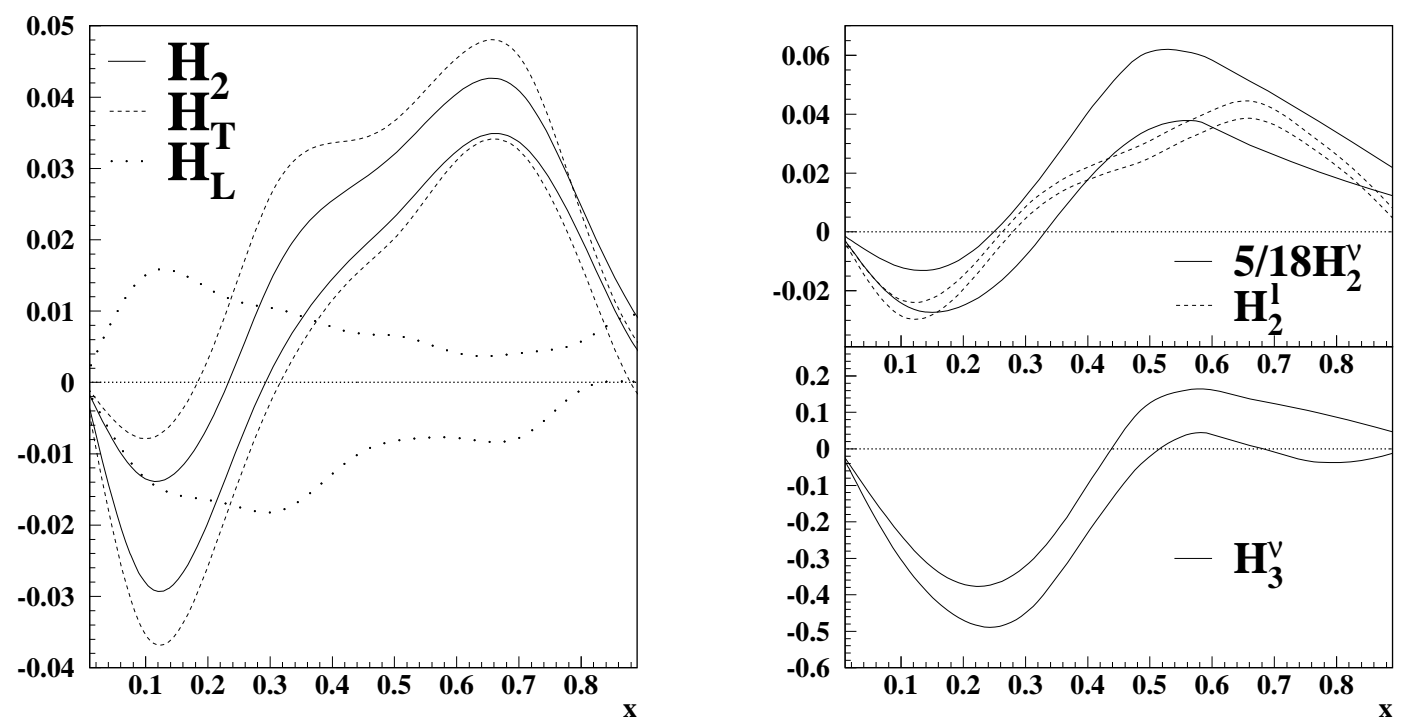

FIGURE 2. Left figure: the $1 \sigma$ error bands for the high-twist terms in the isospin-symmetric combinations of different structure functions (solid lines: $F_{2}$, dashes: $F_{\mathrm{T}}$, dots: $F_{\mathrm{L}}$ ) for charged leptons. Right figure: corresponding $1 \sigma$ bands for neutrino scattering off an isoscalar target (upper panel: $F_{2}$, lower panel: $x F_{3}$ ). The predictions for $F_{2}$ from charged leptons rescaled by the corresponding leading twist terms are also shown for comparison.

certain discrepancy, which is directly translated into a fake twist- 6 contribution, if such term is fitted as well. The values of inelasticity $y$ are large for SLAC data at the highest $Q$ and small for the BCDMS data at the lowest $Q$. For this reason, the discrepancy affects mainly $F_{\mathrm{T}}$, which is more sensitive to $y$ than $F_{2}$. On the other hand the SLAC/BCDMS inconsistency at $Q^{2} \sim 7 \mathrm{GeV}^{2}$ is not crucial for the determination of twist-4 terms. If we rescale the uncertainties of data in this region to bring the pulls at the level of $1 \sigma$, we observe a negligible increase in the corresponding HT errors. Evidently, HT terms are driven by data at the lowest $Q$ available, so that in principle the SLAC/BCDMS data around $Q^{2} \sim 7 \mathrm{GeV}^{2}$ can even be dropped without any loss of statistical power. Preliminary data from the experiment JLAB-E-118 [13] agree with the SLAC data at low $Q$, thus favouring the reliability of the latter at $Q \sim 1 \mathrm{GeV}$, regardless of potential problems in the region of overlap with BCDMS.

Coming to the conclusion that the twist- 6 terms observed are just an artefact due to certain inconsistencies in the data, we drop them from the final version of the fit. This results in a value of $\chi^{2} / \mathrm{NDP}$ of $3815 / 3076=1.25$, which is higher than unity in consideration of the data discrepancies discussed above. Nonetheless the problematic data points are spread out more or less randomly over kinematics and they do not bias the results of the fit. A rescaling of the uncertainties in the data points with the largest pulls, such that the overall $\chi^{2}$ /NDP becomes unity, leads to a modest increase in the errors of PDFs and HTs within 20\%. Figure 2 (left) shows the twist-4 terms obtained in the final version of our fit. The HT contribution to $F_{T}$ is remarkably similar to the one in $F_{2}$, despite the two terms were fitted independently. As a result the HT term in $F_{L}$ defined as $H_{\mathrm{L}}=H_{2}-H_{\mathrm{T}}$ is well comparable to zero within the uncertainties. In the 
final version of the fit we then impose the constraint $H_{2}=H_{T}$ for the isospin-symmetric combinations of structure functions.

Our results indicate the HT contribution to the structure function $R=\sigma_{\mathrm{L}} / \sigma_{\mathrm{T}}$ is also small in the whole range of $x$ considered. This is in contrast to the conclusion of Ref.[2] about a large HT contribution to $R$. We explain such difference by the fact that the low- $Q$ part of the SLAC data was not considered in Ref.[2]. The extrapolation of those results to the lower $Q$ value must be in disagreement with data. In Fig. 3 (left) we compare our predictions for $R$ with the empirical parameterization $R_{\text {SLAC }}$ from SLAC data [3]. The latter is consistent with our calculation based upon the fit with fake twist- 6 terms. This indicates the $R_{\text {SLAC }}$ parameterization is the result of the same inconsistency between SLAC and BCDMS data we discussed above.

It is interesting to determine the HT contributions to (anti)neutrino structure functions independently from the ones extracted from charged lepton data. Due to the structure of the weak Charged Current (CC) some similarities with charged leptons could be expected for $F_{2}$ and $F_{T}$. Since the target nucleon is mostly isoscalar for neutrino data we impose the constraint $H_{2}=H_{T}$, consistently with charged leptons 2 . Figure 2 (right) summarizes our observations. The ratio $H_{2}^{\tau 4} / F_{2}^{\mathrm{LT}}$ is remarkably similar for both (anti)neutrinos and charged leptons over the entire $x$ range. In addition, the use of neutrino data allows us to extract simultaneously the HT contribution to $x F_{3}$. Our results indicate overall $\mathrm{H}_{3}$ provides a negative contribution to the Gross-Llewellyn-Smith integral, which is consistent with the predictions of Ref. [14].

The total contribution of the HT terms into the DIS cross section turns out to be small compared to the leading-twist (LT) part. For a realistic DIS kinematics the ratio of the HT and LT terms is $\lesssim 10 \%$, which justifies the use of the twist expansion in our analysis.

Finally, our results demonstrate high twist contributions to unpolarized structure functions do not vanish in the NNLO approximation. Indeed we find no strong dependence upon the order of QCD calculation used in the leading twist.

\section{IMPACT ON LEADING TWIST}

The use of additional data at low $Q$ values allows to achieve a better separation between leading and higher twists by exploiting their different $Q^{2}$ dependence. The correlation coefficients are indeed substantially reduced in the whole $x$ range by extending the lower cutoff from $2.5 \mathrm{GeV}^{2}$ to $1.0 \mathrm{GeV}^{2}$ and do not exceed 0.3 . This in turn results in reduced theoretical uncertainties.

Our value of $\alpha_{\mathrm{S}}\left(M_{Z}\right)=0.1128 \pm 0.0011$ is consistent with the one obtained in Ref.[9] with the cut $Q^{2}>2.5 \mathrm{GeV}^{2}$ and is in good agreement with the result from the non-singlet DIS data analysis [15]. The PDFs obtained in the fit with the low- $Q$ DIS data included are also close to the ones of Ref.[9]. This manifests a good separation of the LT and

\footnotetext{
2 This relation does not hold in general since the presence of an axial-vector current introduces significant HT contributions to $F_{L}$ in the limit of small $x$ values and vanishing $Q$ (PCAC). This will be discussed in the following. However, we are here focused on a kinematic region in which such effects are marginal $(x \geq 0.045$ and $Q>1.0 \mathrm{GeV})$.
} 

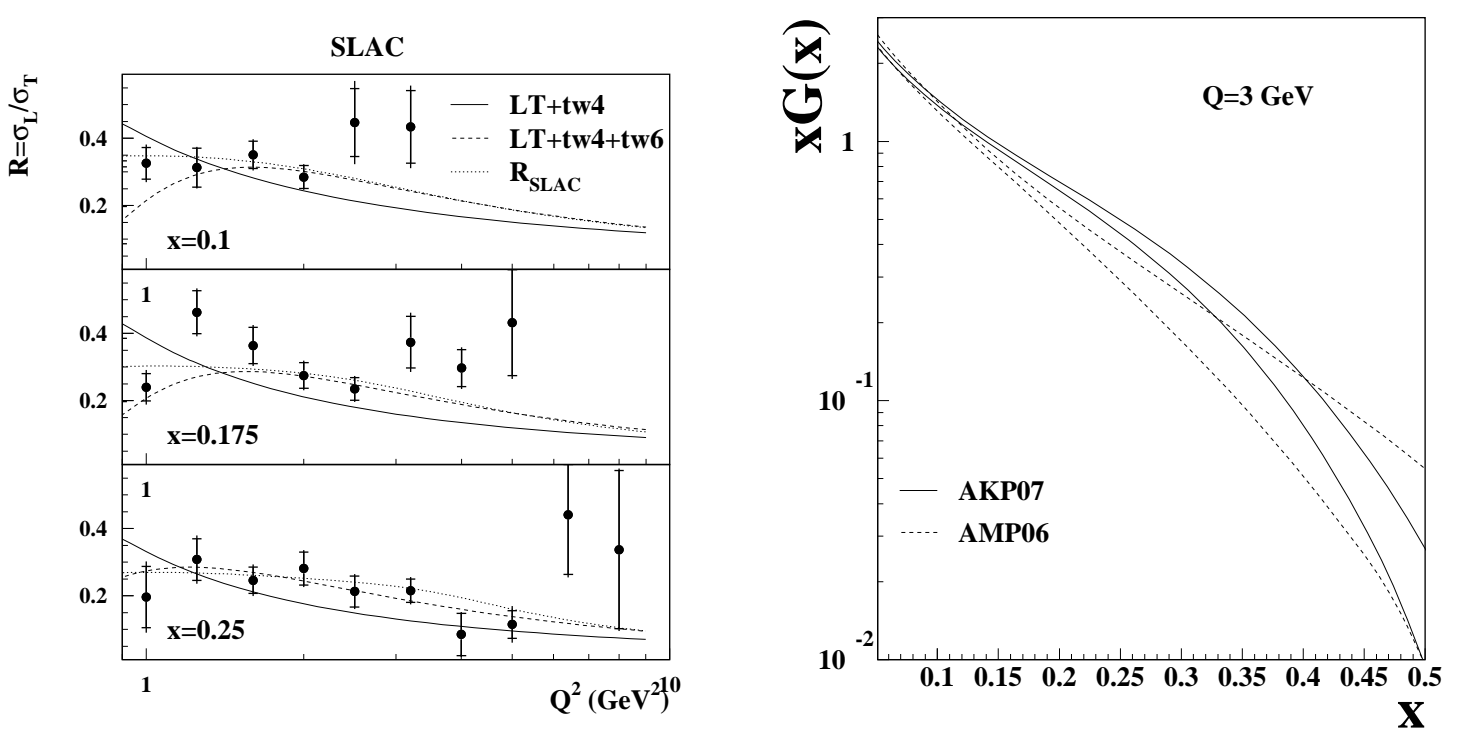

FIGURE 3. Left figure: comparison of the calculations for $R=\sigma_{L} / \sigma_{T}$ including high twist contributions with the empirical parameterization of SLAC data $R_{\text {SLAC }}[3]$. The data points represent SLAC data. Right figure: the $1 \sigma$ error bands for the gluon distribution obtained in our fit (solid lines) compared to one of Ref. [9](dashes).

HT terms in the fit and a stability of the perturbative calculation. The most significant change is observed in the gluon distribution at $x \sim 0.3$, which is enhanced in the low$Q$ fit (see Fig.3 right). This is due to the significant twist-4 term appearing in $R$ in the fit with $Q^{2}>2.5 \mathrm{GeV}^{2}$, similarly to the analysis of Ref.[2]. The LT terms in $R$ is then correspondingly suppressed. Since the LT in $R$ is proportional to the value of the gluon distribution, the latter is also suppressed as a result.

The uncertainties on PDFs are improved as compared to the fit of Ref.[9]. In particular, the $d$-quark distribution is now determined within few per cent at $x \sim 0.2$, which is comparable to the precision of the $u$-quark distribution. This improvement has important phenomenological consequences for the extraction of the weak mixing angle from neutrino data. For instance the analysis by the $\mathrm{NuTeV}$ collaboration [16], based on the Paschos-Wolfenstein relation, requires a good knowledge of the valence distributions in order to guarantee an accurate correction for the target non-isoscalarity [17]. This correction is proportional to the ratio $x_{1} / x_{0}$, where $x_{1}$ and $x_{0}$ are the integrals over $x$ of the iso-vector and iso-scalar combinations of the valence quarks, respectively. From our fit we obtain $x_{1} / x_{0}=0.424(6)$, which provides an uncertainty on the non-isoscalarity of the target comparable to the experimental uncertainties from $\mathrm{NuTeV}$ data.

\section{EXTRAPOLATION TO $Q<1$ GEV}

In order to describe the structure functions in the region of low momentum transfer we apply a smooth interpolation between the high $Q^{2}$ regime, which is described in QCD in terms of LT and HT contributions as discussed above, and the $Q^{2} \rightarrow 0$ predictions 

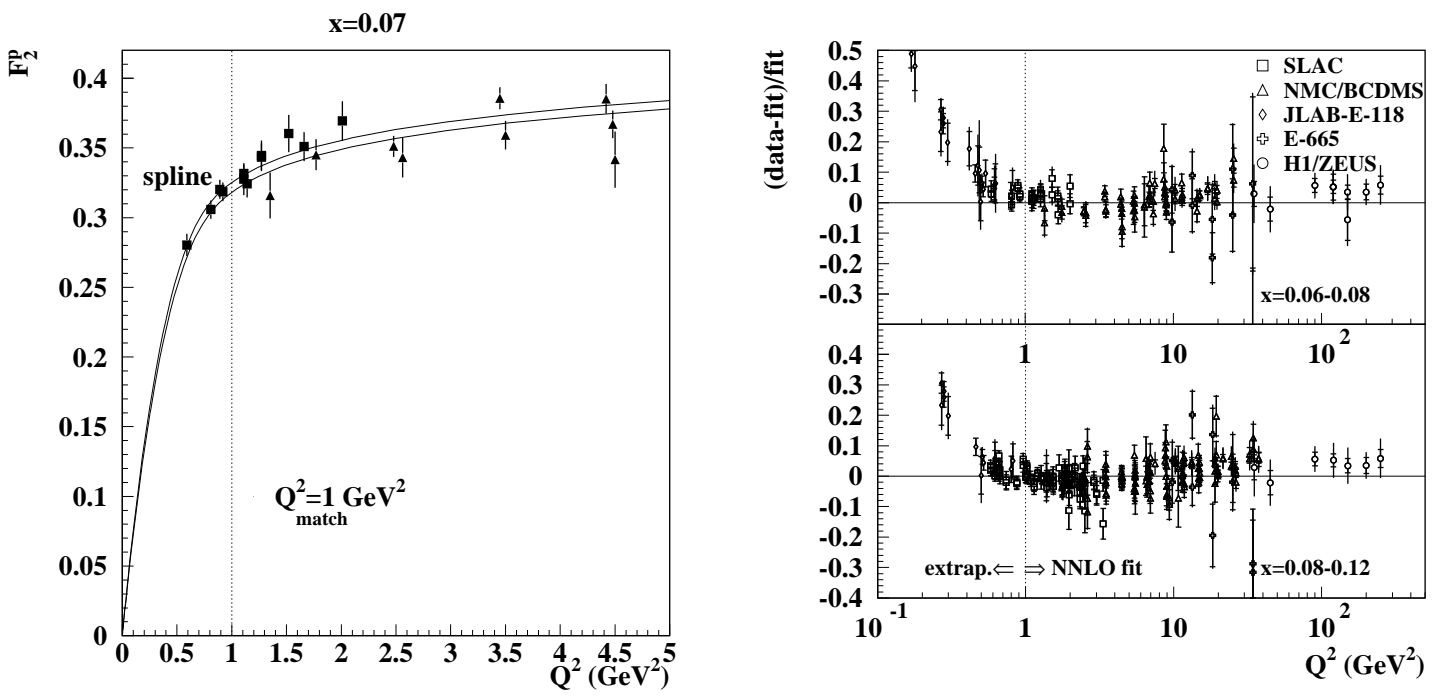

FIGURE 4. Left figure: interpolation of structure functions in the region $0<Q^{2}<1 \mathrm{GeV}^{2}$. The example given in the plot refers to $F_{2}$ for charged lepton scattering on protons at $\mathrm{x}=0.07$ (see text for details). Right figure: pulls with respect to the calculations from our fit for charged leptons as a function of $Q^{2}$. The region $Q^{2}<1 \mathrm{GeV}^{2}$ and data from JLab E-118 were not included in the fit.

derived from current conservation arguments. We use $Q_{m}^{2}=1 \mathrm{GeV}^{2}$ as the matching point between high- and low- $Q^{2}$ regimes.

The conservation of the vector current (CVC) suggests that for the electromagnetic interaction in the limit of $Q^{2} \rightarrow 0$ the structure function $F_{T}$ vanishes as $Q^{2}$ while $F_{L}$ vanishes faster than $F_{T}$ in such a way that $F_{L} / F_{T} \sim Q^{2}$. Although these asymptotic conditions define the value of structure functions at $Q^{2}=0$, they do not specify at which scale such behaviour sets in. On the other hand, at $Q_{m}^{2}$ we know precisely the value of SFs from the twist expansion. In the region $0<Q^{2}<Q_{m}^{2}$ we interpolate by using cubic splines calculated for fixed $x$ values. The corresponding coefficients are fully determined by the condition both functions and derivatives should match with the twist expansion at $Q_{m}^{2}$. Figure 4 (right) illustrates the interpolation procedure for $F_{2}$ on protons in charged lepton scattering.

We obtain a good description of charged lepton data down to $Q^{2} \sim 0.5 \mathrm{GeV}^{2}$, as can be seen from Fig. 4 (left) showing pulls as a function of $Q^{2}$ at a fixed $x$ value. Below that some deviations are visible in the recent JLab E-118 data. This may indicate the transition from dynamics to the behaviour dictated by gauge invariance is slower than in our simple extrapolation of the twist expansion.

In the low- $Q$ region (anti)neutrino cross sections are dominated by the longitudinal structure function $F_{L}$ and the latter is driven by the axial-current interactions. Similarly to the charged lepton case, the structure function $F_{T}$ vanishes as $Q^{2}$ at low $Q^{2}$. This behaviour holds for both the vector and the axial-vector contributions. However, in the longitudinal channel the low- $Q$ behavior of the vector and axial-vector parts are different. The vector component $F_{L}^{\mathrm{V}}$ still vanishes as $Q^{4}$ at low $Q^{2}$ values. 

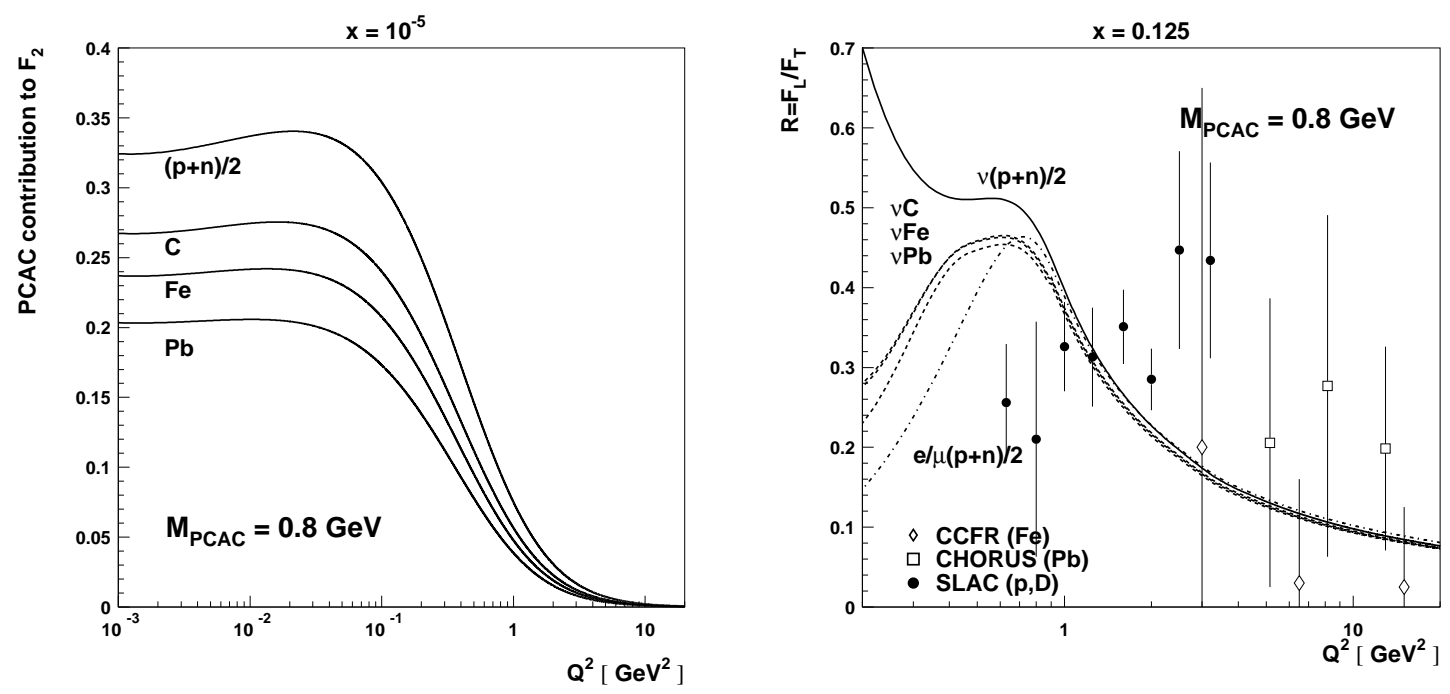

FIGURE 5. Left figure: the PCAC contribution to the neutrino structure function $F_{2}$ calculated for $x=10^{-5}$ as a function of $Q^{2}$ for a few different targets (labels on the curves). A value $M_{\mathrm{PCAC}}=0.8$ $\mathrm{GeV}$ is assumed. Right figure: comparison of the ratio $R=F_{L} / F_{T}$ calculated for the isoscalar nucleon for the charged lepton (dashed-dotted line) and neutrino (solid line) cases at $x=0.125$. Also shown are the results for different nuclear targets $\left({ }^{12} \mathrm{C},{ }^{56} \mathrm{Fe}\right.$ and ${ }^{207} \mathrm{~Pb}$ from top to bottom). A value $M_{\mathrm{PCAC}}=0.8 \mathrm{GeV}$ is assumed. Determinations from SLAC [3], CCFR [19] and CHORUS [12] are given for comparison.

In contrast to the vector current, the axial-vector current is not conserved. For low momentum transfer the divergence of the axial-vector current is proportional to the pion field (Partially Conserved Axial Current or PCAC): $\partial A^{ \pm}=f_{\pi} m_{\pi}^{2} \varphi^{ \pm}$where $m_{\pi}$ is the pion mass, $f_{\pi}=0.93 m_{\pi}$ the pion decay constant and $\varphi^{ \pm}$the pion field in the corresponding charge state. We introduce explicitely a PCAC contribution to $F_{L}^{\mathrm{A}}$ :

$$
F_{L}^{\mathrm{A}}=\gamma^{3} F_{L}^{\mathrm{PCAC}} f_{\mathrm{PCAC}}\left(Q^{2}\right)+\widetilde{F}_{L}^{\mathrm{A}}
$$

where $\gamma=\left(1+4 x^{2} M^{2} / Q^{2}\right)^{1 / 2}, F_{L}^{\mathrm{PCAC}}=f_{\pi}^{2} \sigma_{\pi} / \pi$ and $\sigma_{\pi}=\sigma_{\pi}\left(s, Q^{2}\right)$ is the total cross section for the scattering of a virtual pion with the center-of-mass energy squared $s$. The last term $\widetilde{F}_{L}^{\mathrm{A}}$ is similar to $F_{L}^{\mathrm{V}}$ and vanishes as $Q^{4}$. Since the PCAC contribution is expected to vanish at high $Q^{2}$ we introduce a form factor $f_{\mathrm{PCAC}}\left(Q^{2}\right)=$ $\left(1+Q^{2} / M_{\mathrm{PCAC}}^{2}\right)^{-2}$ [18], where the dipole form is motivated by meson dominance arguments 3 . It is important to note the pion pole does not directly contribute to structure functions and hence the mass scale controlling the PCAC mechanism, $M_{\text {PCAC }}$, cannot be the pion mass itself, but is rather related to higher mass states like $a_{1}, \rho \pi$ etc. This scale is not well known from theory and must be determined with data themselves. A value $M_{\mathrm{PCAC}}=0.8 \mathrm{GeV}$ seems to provide the best agreement with existing low- $Q$ data.

${ }^{3}$ Formally the PCAC contribution can be considered as a high twist term. 

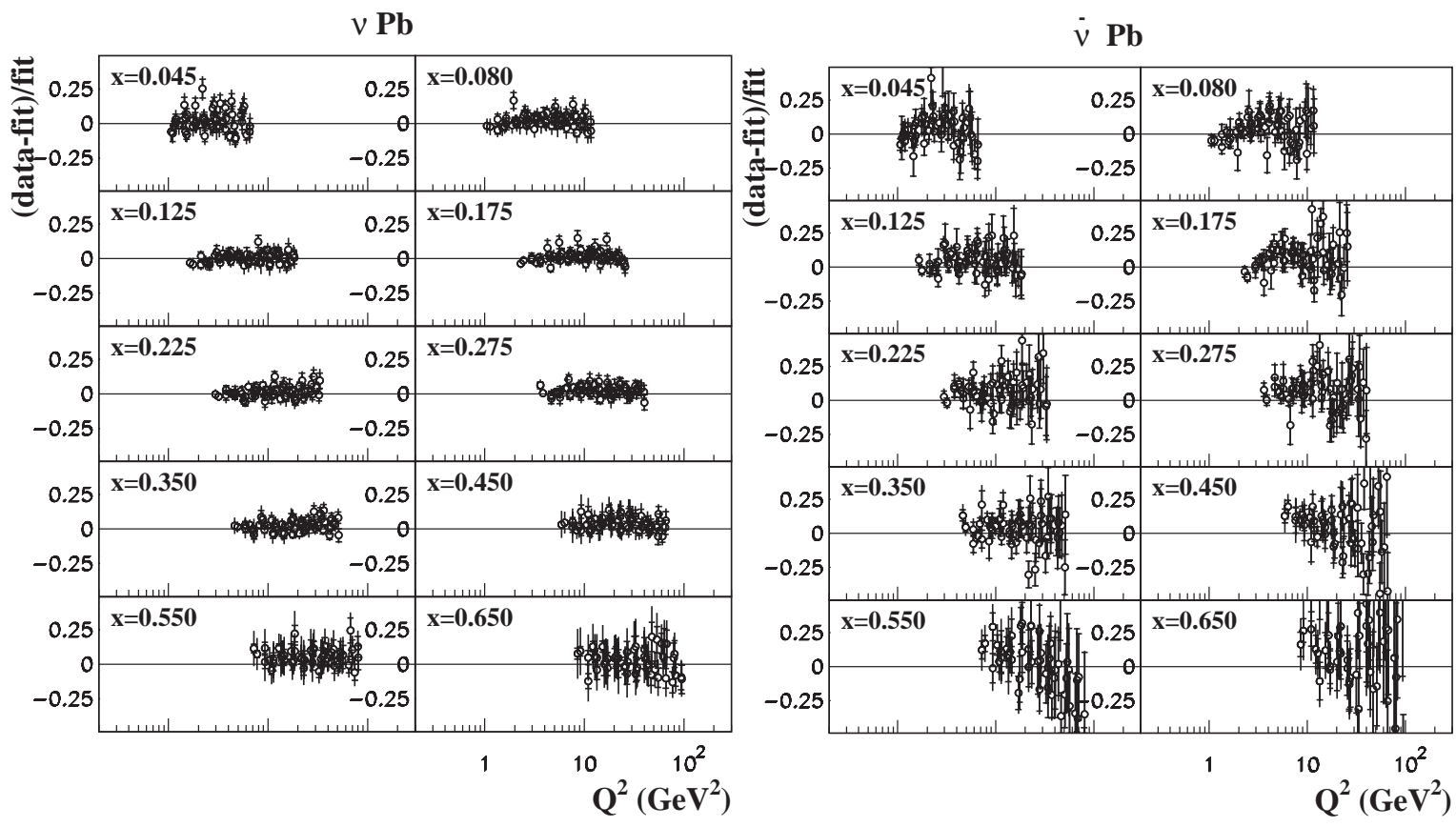

FIGURE 6. Pulls for neutrino (left) and antineutrino (right) cross-section data from CHORUS [12] with respect to our calculations as a function of $Q^{2}$. Nuclear corrections [11] for the lead target and electroweak corrections [21] are taken into account.

Since $F_{2}=\left(F_{L}+F_{T}\right) / \gamma$ the presence of the PCAC terms implies the structure function $F_{2}$ at low $Q^{2}$ is dominated by the nonvanishing $F_{L}^{\mathrm{PCAC}}$ term. Figure 5 (left) illustrates the magnitude of such contribution to $F_{2}$ for an isoscalar nucleon and for a few nuclear targets [18]. The values of $F_{2}$ for heavy nuclear targets are systematically smaller because of nuclear shadowing effect for the pion cross section. Our predictions for the asymptotic value are consistent with the determination $F_{2}\left(Q^{2}=0\right)=0.210 \pm 0.002$ by the CCFR experiment on an iron target [20].

It is instructive to compare the low- $Q^{2}$ behaviour of $R=F_{L} / F_{T}$ for charged-lepton and neutrino scattering. In both cases $F_{T} \propto Q^{2}$ as $Q^{2} \rightarrow 0$. However, if $F_{L} \propto Q^{4}$ for the electromagnetic current, for the weak current $F_{L} \rightarrow F_{L}^{\mathrm{PCAC}}$ and thus $F_{L}$ does not vanish in the low- $Q^{2}$ limit. Then the behavior of $R$ at $Q^{2} \ll 1 \mathrm{GeV}^{2}$ is substantially different for charged-lepton and neutrino scattering. In Figure 5 (right) we illustrate this effect by calculating $R$ as a function of $Q^{2}$ at a fixed $x$ for an isoscalar nucleon and a number of nuclei. We observe the nuclear correction partially reduces the differences between charged leptons and neutrinos and smoothes out the divergence of $R$ in the latter case.

Figure 6 shows the pulls of our fit to neutrino and antineutrino inclusive inelastic cross-section data from the CHORUS experiment [12] on a lead target. The calculation includes nuclear corrections [11] for the lead target, electroweak corrections [21] and the PCAC contribution.

The determination of LT and HT terms is performed from all available data with $Q^{2}>$ $1.0 \mathrm{GeV}^{2}$ and $W>1.8 \mathrm{GeV}$. It is interesting to check the extrapolation of DIS structure functions into the resonance region for $W<1.8 \mathrm{GeV}$. Our results are consistent with the 
duality principle and the integral of the difference between the recent JLab resonance data and the average DIS predictions is consistent with zero within few percent in the entire kinematic region. This can be also considered as an indirect indication in favour of a negligible twist- 6 contribution to structure functions.

\section{ACKNOWLEDGMENTS}

This work is partially supported by the RFBR grant 06-02-16659 and the Russian Ministry of Science and Education Nsh 5911.2006. R.P. thanks USC for supporting this research.

\section{REFERENCES}

1. S. Moch, J. A. M. Vermaseren and A. Vogt, Nucl. Phys. B 688, 101 (2004); A. Vogt, S. Moch and J. A. M. Vermaseren, Nucl. Phys. B 691, 129 (2004); J. A. M. Vermaseren, A. Vogt and S. Moch, Nucl. Phys. B 724, 3 (2005); S. Moch and M. Rogal, arXiv:0704.1740 [hep-ph].

2. J. L. Miramontes, M. A. Miramontes and J. Sanchez Guillen, Phys. Rev. D 40, 2184 (1989).

3. L. W. Whitlow et al., Phys. Lett. B 250, 193 (1990).

4. M. Virchaux and A. Milsztajn, Phys. Lett. B 274, 221 (1992).

5. U. K. Yang and A. Bodek, Eur. Phys. J. C 13, 241 (2000).

6. S. I. Alekhin, Phys. Rev. D 68, 014002 (2003).

7. A. L. Kataev, G. Parente and A. V. Sidorov, Nucl. Phys. B 573, 405 (2000).

8. O. Nachtmann, Nucl. Phys. B 63, 237 (1973); H. Georgi and H. D. Politzer, Phys. Rev. D 14, 1829 (1976).

9. S. Alekhin, K. Melnikov and F. Petriello, Phys. Rev. D 74, 054033 (2006).

10. M. R. Adams et al. [E665 Collaboration], Phys. Rev. D 54, 3006 (1996).

11. S. A. Kulagin and R. Petti, Nucl. Phys. A 765, 126 (2006).

12. G. Önengüt et al. [CHORUS Collaboration], Phys. Lett. B 632, 65 (2006).

13. C. Keppel, private communication.

14. V. M. Braun and A. V. Kolesnichenko, Nucl. Phys. B 283, 723 (1987).

15. J. Blümlein, H. Bottcher and A. Guffanti, Nucl. Phys. B 774, 182 (2007).

16. G. P. Zeller et al. [NuTeV Collaboration], Phys. Rev. Lett. 88, 091802 (2002) [Erratum-ibid. 90, $239902(2003)]$.

17. S. A. Kulagin, Phys. Rev. D 67, 091301 (2003).

18. S. A. Kulagin and R. Petti, arXiv:hep-ph/0703033.

19. U. K. Yang et al. [CCFR/NuTeV Collaboration], Phys. Rev. Lett. 87, 251802 (2001).

20. B. T. Fleming et al. [CCFR Collaboration], Phys. Rev. Lett. 86, 5430 (2001).

21. A. B. Arbuzov, D. Yu. Bardin and L. V. Kalinovskaya, JHEP 0506:078 (2005). 